\title{
Atuação, desafios e interfaces da vigilância em saúde: uma revisão integrativa
}

Health surveillance performance, challenges and interfaces: an integrative review

Desempeño, desafíos y interfaces de la vigilancia en salud: una revisión integradora

Arthur Henrique Resende Porto ${ }^{1 *}$, Gabriela Rodrigues Rezende ${ }^{1}$, Giovanna Ferreira Monfredini ${ }^{1}$, Mariana Barbosa Goulart ${ }^{1}$, Mariana Cunha Ferreira ${ }^{1}$, Olívia Cristina Alves Lopes ${ }^{1}$.

\section{RESUMO}

Objetivo: Reunir informações sólidas e atuais a respeito da importância da vigilância em saúde e suas áreas de atuação e interfaces, apresentando, também, discussão no que concerne a seus principais desafios. Métodos: Trata-se de uma revisão integrativa pautada na busca online nas bases de dados: BVS, PubMed e SciELO. Foram feitas pesquisas separadamente em inglês e em português, usando-se os descritores "public health", "surveillance", "performance", "vigilância em saúde" e "atuação". Foram inclusos artigos publicados entre 2015 e 2020, disponíveis na íntegra e que apresentaram a vigilância como foco central da discussão. Resultados: A amostra foi composta por 17 artigos. Verificou-se que a vigilância é fundamental para os sistemas de saúde, com suas divisões atuando, cada qual em sua área, na identificação, minimização e eliminação de potenciais riscos à saúde, bem como no diagnóstico precoce, caso esses riscos já tenham se convertido em problemas reais. Considerações finais: Com o escopo de alcançar a excelência, deve-se não apenas conhecer a história e atuação e interfaces da vigilância em saúde, mas também enfrentar seus desafios, como aqueles identificados nesse trabalho, se fazendo necessário um constante levantamento bibliográfico acerca das temáticas que permeiam o assunto.

Palavras-chave: Saúde pública, Vigilância, Atuação.

\begin{abstract}
Objective: To gather solid and current information about the importance of health surveillance and its areas of operation and interfaces. It is also presented a discussion regarding its main challenges. Methods: It is an integrative review based on the online search in the databases: VHL, PubMed and SciELO. Research was carried out separately in English and Portuguese, using the descriptors "public health", "surveillance", "performance", "vigilância em saúde" and "atuação". Articles published between 2015 and 2020, full available and whose central focus of the discussion was surveillance, were included. Results: The sample consisted of 17 articles. It was found that surveillance is essential for health systems, with their divisions acting, each in their own area, in the identification, minimization and elimination of potential health risks, as well as in the early diagnosis, if these risks have already been converted into real problems. Final considerations: In order to achieve excellence, it is necessary not only to know the history and performance of health surveillance, but also to face its challenges, such as those identified in this work, making necessary to carry out a constant bibliographical survey on the themes that permeate the subject.
\end{abstract}

Key words: Public health, Surveillance, Performance.

1 Universidade do Estado de Minas Gerais (UEMG), Passos - MG.

*E-mail: arthurporto97@gmail.com 


\section{RESUMEN}

Objetivo: Reunir información sólida y actual sobre la importancia de la vigilancia en salud y sus áreas de actuación y interfaces, presentando también discusión sobre sus principales desafíos. Métodos: Es una revisión integradora basada en la búsqueda online en las bases de datos: BVS, PubMed y SciELO. La investigación se llevó a cabo por separado en inglés y portugués, utilizando los descriptores "public health", "surveillance", "performance", "vigilância em saúde" y "atuação". Se incluyeron artículos publicados entre 2015 y 2020, completamente disponibles y que incluían vigilancia como eje central de la discusión. Resultados: La muestra fue compuesta por 17 artículos. Se constató que la vigilancia es fundamental para los sistemas de salud, con sus divisiones actuando, cada una en su propio ámbito, en identificación, minimización y eliminación de potenciales riesgos para la salud, así como en diagnóstico precoz, caso estos riesgos ya se han convertido en problemas reales. Consideraciones finales: Para alcanzar excelencia, es necesario no solo conocer la historia y desempeño de la vigilancia en salud, pero también enfrentar sus desafíos, como los identificados en este trabajo, por lo que es necesario realizar un constante relevamiento bibliográfico sobre las subtematicas que impregnan el assunto.

Palabras clave: Salud pública, Vigilancia, Actuación.

\section{INTRODUÇÃO}

A Vigilância em Saúde é considerada um eixo essencial da saúde pública, e as práticas de vigilância em saúde, tais quais se conhecem hoje, remontam a experiências históricas datadas dos séculos XVIII e XIX, como a medicina de estado da Alemanha, a medicina urbana da França e a medicina social da Inglaterra, dentre outros estudos que ocorreram, aproximadamente, na mesma época. No Brasil, apesar de as primeiras ações datarem do fim do século XIX, o modelo de vigilância em saúde surgiu nas décadas de 1980-90 como uma alternativa aos modelos de atenção à saúde até então hegemônicos, propondo práticas interdisciplinares que incorporassem diferentes saberes (NETO JHVS, et al., 2020).

A Constituição Federal de 1988 determina, em seu artigo 200, inciso II, que uma das atribuições do Sistema Único de Saúde (SUS) consiste em "executar as ações de vigilância sanitária e epidemiológica, bem como as de saúde do trabalhador". Por sua vez, a lei 8080/90, em seu artigo 15, inciso VII, complementa que cabe às diversas esferas governamentais a "participação de formulação da política e da execução das ações de saneamento básico e colaboração na proteção e recuperação do meio ambiente", incluindo, dessa forma, a vigilância ambiental como parte do SUS (COSTA ILOF, et al., 2020).

Depreende-se, do exposto acima, as atividades de vigilância em saúde são parte fundamental do sistema público de saúde vigente no Brasil, contribuindo, portanto, para a qualidade de vida de todos os cidadãos residentes no país, no entanto, uma parcela desses não faz uso dos serviços assistenciais do SUS. As ações de vigilância devem integrar todas as Regiões de Saúde estabelecidas em território nacional, como regulamenta o decreto 7508, de 2011 (TEIXEIRA MG, et al., 2018).

A Política Nacional de Vigilância em Saúde (PNVS), instituída em 2018 por meio da resolução ํo 588 do Conselho Nacional de Saúde, define vigilância como um processo sistemático incessante no qual se coletam e analisam dados de eventos relevantes sobre a saúde, com o escopo de organizar e aperfeiçoar medidas de saúde pública e, consequentemente, potencializar a promoção da saúde, prevenção e redução de riscos e danos para a sociedade (BRASIL, 2018).

Dentre as competências para a estruturação da vigilância em saúde, pode-se destacar: integração e articulação entre as vigilâncias a articulação entre as vigilâncias, bem como articulação com a rede de atenção à saúde; os sistemas de informação integrados; a gestão do processo de trabalho; a educação permanente; os estudos e pesquisas; a comunicação e o controle social, associado à regionalização das ações e serviços de vigilância em saúde. Todas essas competências são consideradas essenciais para informar as mudanças nas políticas, orientar as novas intervenções do programa, aprimorar as comunicações públicas e ajudar as agências a avaliar os investimentos em pesquisa (ALBURQUERQUE AC, et al., 2019). 
Nesse sentido, o presente artigo consiste em uma revisão integrativa acerca da atuação da vigilância em saúde, e tem como objetivo reunir informações sólidas e atuais a respeito de sua importância e áreas de atuação, apresentando, também, discussão no que concerne a seus principais desafios e interfaces.

\section{MÉTODOS}

Trata-se de uma revisão integrativa de literatura pautada na busca online em bases de dados de divulgação do conhecimento técnico e científico da área da saúde: Biblioteca Virtual em Saúde (BVS), PubMed e SciElo.

Primeiramente, realizou-se uma seleção de descritores de assunto que orientassem as pesquisas em inglês e português. Assim, foram utilizados neste estudo os descritores "Public health", "Surveillance", ambos como componentes do título e "performance" como parte do resumo, para a busca em inglês. Contudo, para as buscas em português utilizaram-se os descritores "Vigilância em saúde" no título e "atuação" no resumo.

Com base na pesquisa com os descritores em inglês, anteriormente citados, foram encontrados 27 documentos na base BVS, seis na SciElo e 27 na base PubMed, totalizando 60 artigos. Foram utilizados como critérios de inclusão artigos publicados a partir de 2015, a disponibilidade do documento completo, além de limitar as bases em LILACS, Medline e Fiocruz, reduzindo os resultados totais para 22 artigos, sendo 14 na BVS, dois SciElo e seis PubMed.

De modo semelhante, realizou-se a pesquisa com os descritores em português, obtendo como resultados iniciais 27 estudos na BVS, 25 na SciElo e nenhum na PubMed, isto é, um total de 52 artigos. Ao aplicaremse os filtros, houve redução para um total de 32 documentos (15 BVS, 17 SciElo). Assim, foram selecionados 54 artigos para uma nova fase de avaliação.

Dos 54 estudos previamente selecionados, 25 foram excluídos devido à repetição nas bases pesquisadas ou por serem descritos como monografias. Logo, 29 artigos foram escolhidos para a próxima etapa de análise, baseada nos resumos.

Nesse ciclo, houve concordância dos autores, após criteriosa leitura e verificação dos resumos, em excluir estudos com enfoque que fugia às atuações do serviço de vigilância, com direcionamento intenso nas moléstias e pouca descrição do serviço. Após essa seleção, restaram 17 artigos a serem incluídos nesta revisão (Figura 1).

Figura 1 - Fluxograma descrevendo o processo de busca e seleção dos artigos.

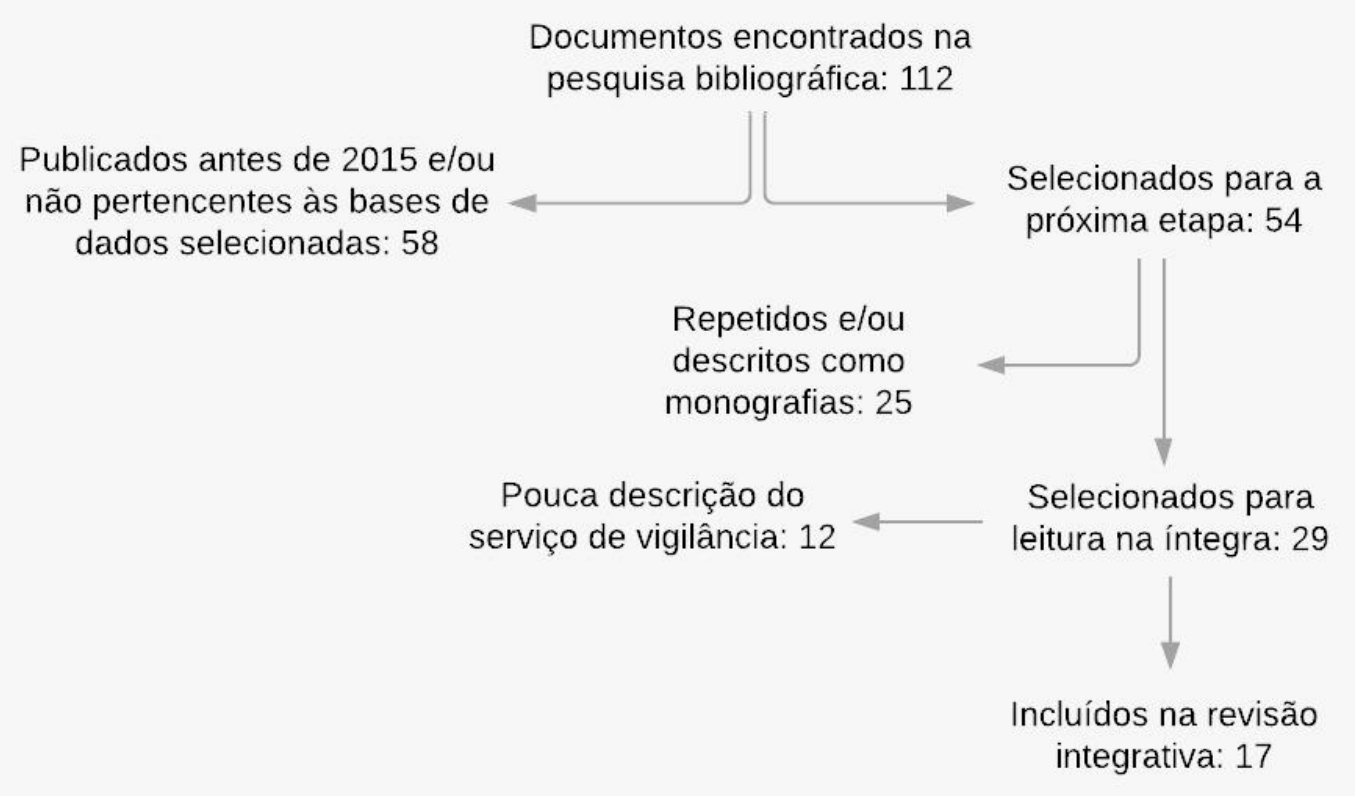

Fonte: Porto AHR, et al., 2021. 
Ao longo do trabalho, com o escopo de abordar quesitos legais, foram acrescentados dois documentos oficiais e/ou secundários de centros formadores, devido à pertinência com o propósito do estudo. Deste modo foi possível basear a revisão na realidade do sistema de saúde do país, tratando mais precisamente da abordagem da vigilância em saúde no contexto do SUS.

$\mathrm{Na}$ análise dos estudos incluídos foram realizadas comparações das informações disponíveis e complementaridade dos artigos.

\section{RESULTADOS E DISCUSSÃO}

A amostra foi composta por 17 artigos e dois documentos oficiais e/ou secundários de centros formadores. Para melhor sistematização dos resultados dos artigos, estes foram inseridos em um quadro, com: autor/ano, dimensão, periódico, método e principais achados (Quadro 1).

Dos 17 artigos analisados, dez estudos foram realizados no Brasil seguido pelos EUA e pela Inglaterra com dois estudos cada; e Bélgica, Etiópia e Canadá com apenas um estudo. No quesito temporal, houve quatro estudos em 2020, três em 2019, quatro em 2018 cinco em 2017 e um em 2016.

No âmbito da Vigilância em Saúde, observou-se que cinco estudos abordam como temática central a Vigilância ambiental, quatro a Vigilância Sanitária, cinco a Vigilância do Trabalhador e seis a Vigilância Epidemiológica. Dois artigos tiveram influência de mais de uma temática central.

Nesse contexto, a análise dos resultados foi dividida em cinco categorias relevantes de discussão, sendo elas: atuação e desafios da Vigilância Ambiental; atuação e desafios da Vigilância em Saúde do Trabalhador; atuação e desafios da Vigilância Sanitária, atuação e desafios da Vigilância Epidemiológica e impactos gerais. 
Quadro 1 - Distribuição dos artigos nacionais e internacionais selecionados quanto a autor/ano, dimensão, periódico e método.

\begin{tabular}{|c|c|c|c|c|}
\hline $\mathbf{N}$ & Autor/Ano & Dimensão & Periódico & Método \\
\hline 1 & Moimaz SAS, et al., 2020 & $\begin{array}{l}\text { Vigilância Ambiental e } \\
\text { Sanitária }\end{array}$ & Ciência \& Saúde Coletiva & $\begin{array}{l}\text { Exploratório, observacional e quali- } \\
\text { quantitativo }\end{array}$ \\
\hline 2 & Yang L, et al., 2020 & $\begin{array}{l}\text { Vigilância em Saúde do } \\
\text { Trabalhador }\end{array}$ & American Journal of Industrial Medicine & $\begin{array}{c}\text { Exploratório, observacional e } \\
\text { qualitativo }\end{array}$ \\
\hline 3 & Vanden-Berg O, et al., 2018 & Vigilância Epidemiológica & Frontiers in medicine & $\begin{array}{c}\text { Descritivo, observacional e } \\
\text { qualitativo }\end{array}$ \\
\hline 4 & Amorim LA, et al., 2017 & $\begin{array}{l}\text { Vigilância em Saúde do } \\
\text { Trabalhador }\end{array}$ & Ciência \& Saúde Coletiva & $\begin{array}{c}\text { Descritivo, observacional quali- } \\
\text { quantitativo }\end{array}$ \\
\hline 5 & Silveira M e Fenner ALD, 2017 & Vigilância Ambiental & Ciência \& Saúde Coletiva & $\begin{array}{c}\text { Descritivo, observacional e } \\
\text { qualitativo }\end{array}$ \\
\hline 6 & Costa GRT, et al., 2016 & Vigilância Ambiental & Com. em Ciências da Saúde & $\begin{array}{c}\text { Descritivo, observacional e } \\
\text { qualitativo }\end{array}$ \\
\hline 7 & Boulieri A, et al., 2020 & Vigilância epidemiológica & Oxford University Press & $\begin{array}{c}\text { Exploratório, observacional e } \\
\text { qualitativo }\end{array}$ \\
\hline 8 & Maturino MM, et al., 2018 & $\begin{array}{c}\text { Vigilância em Saúde do } \\
\text { Trabalhador }\end{array}$ & Revista Brasileira de Saúde Ocup. & $\begin{array}{c}\text { Descritivo, observacional e } \\
\text { qualitativo }\end{array}$ \\
\hline 9 & Arehart CH, et al., 2019 & Vigilância Epidemiológica & Scientific Reports: nature research & $\begin{array}{c}\text { Exploratório, observacional e } \\
\text { qualitativo }\end{array}$ \\
\hline
\end{tabular}




\begin{tabular}{|c|c|c|c|c|}
\hline $\mathbf{N}$ & Autor/Ano & Dimensão & Periódico & Método \\
\hline 10 & Rivera LA, et al., 2017 & Vigilância Epidemiológica & BMC Public Health & $\begin{array}{c}\text { Exploratório, observacional e } \\
\text { qualitativo }\end{array}$ \\
\hline 11 & Dantas AA e Souza MKB, 2020 & Vigilância Sanitária & Revista Bahiana de Enferm. & $\begin{array}{c}\text { Descritivo, observacional e } \\
\text { qualitativo }\end{array}$ \\
\hline 12 & Amancio MATM, et al., 2017 & $\begin{array}{c}\text { Vigilância em Saúde do } \\
\text { Trabalhador }\end{array}$ & Revista Brasileira de Saúde Ocup. & $\begin{array}{c}\text { Descritivo, observacional e } \\
\text { qualitativo }\end{array}$ \\
\hline 13 & Oldroyd RA, et al., 2018 & Vigilância Sanitária & JMIR Public Health and Surveillan-ce & $\begin{array}{c}\text { Descritivo, observacional e } \\
\text { qualitativo }\end{array}$ \\
\hline 14 & Alemu T, et al., 2019 & Vigilância Epidemiológica & BMC Public Health & $\begin{array}{l}\text { Exploratório, observacional e quali- } \\
\text { quantitativo }\end{array}$ \\
\hline 15 & Pintor EAS e Garbin AC, 2019 & $\begin{array}{c}\text { Vigilância em Saúde do } \\
\text { Trabalhador }\end{array}$ & Revista Brasileira de Saúde Ocup. & $\begin{array}{c}\text { Analítico, observacional e quali- } \\
\text { quantitativo }\end{array}$ \\
\hline 16 & Donateli C, et al., 2017 & $\begin{array}{l}\text { Vigilância Sanitária, } \\
\text { Epidemiológica e Ambiental }\end{array}$ & Associação Brasileira de Saúde Coletiva & $\begin{array}{c}\text { Descritivo, observacional e } \\
\text { qualitativo }\end{array}$ \\
\hline 17 & Souza GS, et al., 2017 & Vigilância Ambiental & Ciência \& Saúde Coletiva & $\begin{array}{c}\text { Descritivo, observacional e } \\
\text { qualitativo }\end{array}$ \\
\hline
\end{tabular}

Fonte: Porto AHR, et al., 2021. 


\section{Atuação e desafios da Vigilância Ambiental}

O termo Vigilância em Saúde Ambiental (VSA) consiste em um:

"conjunto de ações que proporcionam o conhecimento e a detecção de mudanças nos fatores determinantes e condicionantes do meio ambiente que interferem na saúde humana, com a finalidade de identificar as medidas de prevenção e controle dos fatores de risco ambientais relacionados às doenças ou a outros agravos à saúde" (BRASIL, 2018).

Ademais, é sabido que competem à VSA os procedimentos de vigilância epidemiológica, associados a contaminantes ambientais, principalmente aqueles relacionados com a exposição a agrotóxicos, amianto, mercúrio, benzeno e chumbo. Desse modo, as principais áreas de atuação da Vigilância Ambiental são: Vigilância da qualidade da água para consumo humano (VIGIAGUA); Vigilância em saúde de populações expostas a poluentes atmosféricos (VIGIAR); Vigilância em saúde de populações expostas a contaminantes químicos (VIGIPEQ); Vigilância em saúde ambiental relacionada aos riscos decorrentes de desastres (VIGIDESASTRES) e Vigilância em saúde ambiental relacionada aos fatores físicos (VIGIFIS) (SOUZA GS, et al., 2017).

Sabendo das competências e atribuições da VSA no SUS e de acordo com a revisão realizada, depreendese que é dever dessa fiscalizar o teor de agrotóxicos contidos em meio aéreo e aquoso, que interferem direta e indiretamente na saúde da população, haja vista que o uso indiscriminado desses pode causar intoxicação, desenvolvimento de doenças crônicas e até culminar em morte. Contudo, a problemática dos agrotóxicos no contexto da Vigilância em Saúde tem focado tradicionalmente na saúde do trabalhador, solo e água, negligenciando a população consumidora dos produtos agroquímicos. Nesse sentido, impõe-se a necessidade de fomentar o aprimoramento das medidas de VSA para grupos populacionais expostos a produtos contaminados por agrotóxicos (SOUZA GS, et al., 2017).

Ainda no que tange à qualidade da água e suas repercussões na saúde da população, um dos trabalhos selecionados para essa revisão faz uma explanação sobre o teor de fluoretação da água de abastecimento público e a relação com prevenção de cáries dentárias na população, tendo como alvo do estudo a cidade de Barretos-SP. Constatou-se que, após dez anos da implementação desse procedimento no município, $50 \%$ das crianças do grupo etário de sete a 10 anos não apresentavam nenhum dente permanente atacado pela cárie. Já dentre as crianças de três a cinco anos, $51,6 \%$ não possuíam nenhum dente primário acometido (MOIMAZ SAS, et al., 2020).

Com isso, nota-se a relevância da VSA no controle da qualidade da água ofertada à população e, consequentemente, na minimização de doenças associadas a esse recurso. Fica comprovada, portanto, a necessidade de ações que fomentem a prevenção e a monitorização relacionados aos fatores ambientais em geral, tais como os programas de heterocontrole, a fim de cada vez mais resguardar a saúde do ser humano (MOIMAZ SAS, et al., 2020).

Além do exposto, foi observado que, apesar de ações preventivas como as citadas acima serem eficazes, há lacunas quando são analisadas questões como notificação e alimentação das bases de dados das medidas de vigilância adotadas. Como exemplo, deve ser enfatizada a falta de integração entre as diferentes áreas da vigilância. Em vários municípios, não há uma integração sequer em termos de estrutura física, o que favorece a fragmentação do trabalho nos diferentes níveis de atenção (DONATELI C, et al., 2017).

Outro ponto a ser discutido consiste nos determinantes sociais e sua influência com relação à Avaliação de Impactos à Saúde (AIS), isto é, a combinação de procedimentos, métodos e ferramentas que permitem avaliar uma proposta de política, plano ou programa quanto aos seus potenciais impactos à saúde, bem como a ocorrência desses efeitos na população, segundo definição da Organização Mundial de Saúde (OMS). O autor defende que, quando uma AIS é realizada, os determinantes da saúde podem ter influência decisiva para efeito de uma avaliação de uma política, programa ou projeto e, desse modo, esse conjunto de condições denominado determinantes sociais é de suma importância para avaliar as condições relativas a esse aspecto em uma determinada população (SILVEIRA M e FENNER ALD, 2017). 
Assim, é evidente a relevância da atuação da VSA e suas diversas atuações na prevenção e monitoramento de água, ar e alimentos de forma a garantir a saúde da população. Contudo, a partir dos trabalhos expostos, percebem-se desafios quanto à notificação de dados e à fragmentação do sistema, o que juntamente com os determinantes sociais da saúde limitam a atuação da Vigilância (DONATELI C, et al., 2017; SILVEIRA M e FENNER ALD, 2017).

\section{Atuação e desafios da Vigilância Epidemiológica}

De acordo com a lei o 8.080 de 19 de setembro de 1990, Vigilância Epidemiológica (VE) é definida como um conjunto de ações que proporciona o conhecimento, a detecção ou prevenção de qualquer mudança nos fatores determinantes e condicionantes da saúde individual ou coletiva, com a finalidade de recomendar e adotar as medidas de prevenção e controle das doenças ou agravos (BRASIL, 1990).

Nesse sentido, o monitoramento espacial online de tendências na saúde é de grande relevância para a vigilância epidemiológica, sendo usualmente utilizado para compreensão da etiologia dos eventuais problemas de saúde pública e do impacto de intervenções em saúde. Além disso, é essencial que se entendam as tendências das doenças de acordo com as diferentes épocas e localidades, de modo a se identificar os fatores de risco (BOULIERI A, et al., 2020).

Desse modo, de acordo com os trabalhos compilados por esta revisão integrativa, observou-se uma tendência de uso de tecnologias a fim de melhorar o recrutamento de dados. Observa-se, por exemplo, que háum atraso entre o surgimento dos sintomas e as notificações usuais feitas nos sistemas de saúde, o que poderia ser melhorado com o uso da tecnologia em pesquisa online. Contudo, dependendo do nível socioeconômico da localidade, nem todas as pessoas dispõem de acesso à internet, o que causa uma limitação desse sistema de pesquisa de dados, uma vez que este não traz total veracidade dos fatos. Assim, entende-se a pesquisa online de dados epidemiológicos como uma forma complementar, mas que não pode substituir a pesquisa e notificação tradicionais já existentes (AREHART CH, et al., 2019).

Além dos dados já expostos, ressalta-se que a falha no sistema de vigilância epidemiológica também pode ser atribuída a deficiências de comunicação entre os níveis de vigilância, exemplificadas pela inacessibilidade ao computador nas unidades de saúde, à falta de orçamento, à ineficiência de supervisão dos dados, e a dados colhidos incompletos, o que faz com que inúmeras variáveis se tornem incongruentes no sistema, fato que contribui para a falta de veracidade no compilamento dos dados colhidos (ALEMU T, et al., 2019).

Outro ponto relevante a ser ressaltado refere-se à efetividade da padronização de protocolos de vigilância. Um dos estudos abordados nessa revisão coloca em xeque tal premissa, mostrando que muitas vezes tal padronização é prejudicial, pois diversas localidades de um país e até mesmo estado, possuem realidades muito distintas, sendo necessária, portanto, a personalização de protocolos para que estes atinjam a eficácia da coleta de dados na VE (RIVERA LA, et al., 2017).

\section{Atuação e desafios da Vigilância Sanitária}

De acordo com o parágrafo primeiro do artigo 6으 da lei o 8.080 de 19 de setembro de 1990, entende-se por Vigilância Sanitária (VISA) como um:

"conjunto de ações capaz de eliminar, diminuir ou prevenir riscos à saúde e de intervir nos problemas sanitários decorrentes do meio ambiente, da produção e circulação de bens e da prestação de serviços de interesse da saúde" (BRASIL, 1990).

Dessa maneira, hodiernamente, uma das áreas de atuação da VISA, talvez a mais conhecida, é a de vigilância de alimentos. Nesse âmbito, os agentes possuem como atribuição a fiscalização de locais que produzem, transportam e comercializam alimentos, com o objetivo de diminuir riscos à saúde, como é o caso de intoxicação alimentar (OLDROYD RA, et al., 2018).

Nesse contexto, pode-se mencionar a intoxicação alimentar, da qual muitas vítimas se recuperam rapidamente, mas, em populações mais vulneráveis (principalmente aquelas com mais de 65 ou menos de 
cinco anos, mulheres grávidas e pessoas com sistema imune comprometido), pode causar problemas duradouros, além de serem passíveis de culminar em morte. Esse é apenas um exemplo de como a atuação da VISA pode prevenir agravos e, até mesmo, diminuir o número de pessoas à procura de um serviço de saúde, de forma a reduzir ligeiramente a sobrecarga dos serviços (OLDROYD RA, et al., 2018).

Contudo, ainda existem desafios a serem enfrentados, haja vista que as vigilâncias municipais, muitas vezes, ofertam a atenção à saúde de maneira fragmentada. Isso implica em maior dificuldade no cumprimento de metas propostas pelo governo estadual, de modo que a atuação da VISA nos municípios torna-se pouco efetiva, o que comprova a necessidade da integração do sistema (DONATELI C, et al., 2017).

Somado a isso, ressalta-se que manter a situação higiênico-sanitária das unidades de saúde em boas condições é responsabilidade da gestão a nível municipal, distrital e das equipes. Entretanto, muitas questões são negligenciadas, o que pode interferir na saúde dos usuários e no trabalho da equipe. Isso sugere a necessidade de formulação de estratégias articuladas entre a VISA e outros setores que lidam com as unidades de saúde (DANTAS AA e BONFIM MK, 2020).

Assim, conclui-se que a VISA possui atuação diversa, que verifica as condições de bens de consumo, como o caso dos alimentos citados, da prestação de serviços, como o caso das unidades de saúde expostas, e outros. Logo, mostra-se a importância dela na prevenção de agravos e na diminuição da quantidade de pessoas procurando serviços de saúde com sinais e sintomas que poderiam ter sido prevenidos com simples ações de fiscalização (BRASIL, 1990; OLDROYD RA, et al., 2018).

\section{Atuação e desafios da Vigilância da Saúde do Trabalhador}

Segundo o parágrafo terceiro do artigo 6으 da lei no 8.080 de 19 de setembro de 1990, entende-se por Vigilância em Saúde do Trabalhador (VISAT) como um:

"conjunto de atividades que se destina, através de vigilância epidemiológica e vigilância sanitária, à promoção e proteção da saúde dos trabalhadores, assim como visa a recuperação e reabilitação da saúde dos trabalhadores submetidos aos riscos e agravos advindos das condições de trabalho" (BRASIL, 1990).

Nesse contexto, a VISAT mostra-se como o eixo estruturante do cuidado à saúde dos trabalhadores pela Política Nacional de Saúde do Trabalhador e da Trabalhadora (BRASIL, 2012). Ela se organiza em dois componentes básicos: a vigilância dos agravos à saúde relacionados ao trabalho - que se articula com a VE - e a vigilância dos ambientes e condições de trabalho - que se articula com a VISA e a VSA (AMORIM LA, et al., 2017).

Dessa maneira, a VISAT surgiu como uma demanda para garantir a integralidade da atenção à saúde do trabalhador. Além disso, o cuidado à saúde desse deve considerar a inserção dele nos processos produtivos, haja vista que o trabalho é determinante importante no processo saúde-doença (AMORIM LA, et al., 2017). Ademais, o acolhimento deve ser o primeiro vínculo criado entre o profissional da saúde e o profissional a ser atendido, uma vez que ele, muitas vezes, é o responsável pela aderência ao tratamento (AMANCIO MATM, et al., 2017).

No Brasil, a atenção primária é retratada como o principal nível de cuidado à saúde do trabalhador. Contudo, alguns problemas relacionados à atuação desse nível de atenção foram encontrados. Dentre eles: o menor envolvimento das equipes em ações de VISAT à medida que aumenta a complexidade; a grande quantidade de profissionais que desconhecem ou não participam do processo de mapeamento das atividades no território de atuação da equipe; o fato da Estratégia de Saúde da Família não ser reconhecida como um canal de denúncias para problemas relacionados ao trabalho; pouco reconhecimento dos profissionais acerca de trabalhos progressos; baixa notificação de doenças relacionadas ao trabalho ao Sistema de Informação de Agravos de Notificação (SINAN), entre outros (AMORIM LA, et al., 2017).

Esse último mostra um erro grave que ocorre, haja vista que os casos de violência, seja ela relacionada ao trabalho ou não, são de notificação compulsória no SINAN (PINTOR EAS e GARBIN AC,2019). Ministério da Saúde adotou a notificação como estratégia central para reconhecer, combater e prevenir a violência, de 
forma que é um elemento chave na atenção integral às pessoas. Outro erro que pode ocorrer no processo de notificação é o atraso. Isso limita a capacidade dos indicadores de refletir questões emergentes em saúde do trabalhador, de modo que perde a eficácia na orientação de intervenções e práticas oportunas e na medição do progresso e utilidade dos programas da VISAT (YANG L, et al., 2020).

Destarte, a VISAT mostra-se com o objetivo de fiscalizar, educar, sensibilizar, mobilizar os envolvidos, treinar os funcionários e levar a redução de riscos de trabalho. Contudo, muitos desafios precisam ser superados para que essas ações sejam completamente efetivas. Por fim,para que haja efetiva manutenção das medidas de proteção nos ambientes de trabalho, são necessários o conhecimento sobre os riscos ocupacionais e seus efeitos, a organização dos trabalhadores e a defesa da saúde no trabalho (AMANCIO MATM, et al., 2017).

\section{Impactos gerais}

Verificou-se que as diferentes áreas da Vigilância em Saúde são fundamentais para os sistemas de saúde vigentes na contemporaneidade, não somente no Brasil, por meio do SUS, mas também em outros países. Ambas as divisões atuam, cada qual em sua área, na identificação, minimização e eliminação de potenciais riscos à saúde da população, bem como no diagnóstico precoce, caso esses riscos já tenham se convertido em problemas reais. Agem, assim, diretamente nos fatores determinantes e/ou condicionantes do processo saúde-doença, o que contribui para uma medicina preventiva integral e de qualidade (BRASIL, 2018).

Alguns dos desafios mais importantes encontrados foram: atraso ou ausência de notificação nos sistemas de vigilância (o que foi observado em todas as quatro divisões); fragmentação dos serviços ofertados; padronização dos protocolos que não leva em consideração as particularidades locais; falta de integração entre as áreas da vigilância e ausência de treinamento e envolvimento das equipes de Atenção Primária (AMORIM LA, et. al., 2017; DONATELI C, et al., 2017; RIVERA LA, et al., 2017).

\section{CONSIDERAÇÕES FINAIS}

Como se percebeu neste trabalho, as estratégias e os desafios de atuação da Vigilância em Saúde são amplamente discutidos no mundo, de modo a aprimorar as técnicas de notificação e estudos epidemiológicos. Como um país o qual a saúde é pública e universal, como preza o SUS, o Brasil necessita de pesquisas intensas para seguir os melhores caminhos para aperfeiçoar seus departamentos de vigília. Com o escopo de alcançar a excelência, deve-se não apenas conhecer a história e atuação da vigilância em saúde, mas também enfrentar seus desafios, como aqueles identificados nesse trabalho, se fazendo necessário um constante levantamento bibliográfico acerca das temáticas que permeiam o assunto.

\section{REFERÊNCIAS}

1. ALBUQUERQUE, AC. Avaliação de desempenho da regionalização da vigilância em saúde em seis Regiões de Saúde brasileiras. Cadernos de Saúde Pública, 2019; 2(supl.2):e00065218.

2. ALEMU T, et al. Evaluation of public health surveillance system performance in Dangila district, Northwest Ethiopia: a concurrent embedded mixed quantitative/qualitative facility-based cross-sectional study. BMC Public Health, 2019; 19: 1343.

3. AMANCIO MATM, et al. Atenção à saúde do trabalhador de postos de revenda de combustíveis: relato sobre a implantação de programa de vigilância e de estratégia de acolhimento de trabalhadores em Campinas/SP. Revista Brasileira de Saúde Ocupacional, 2017; 42(supl. 1): e11s.

4. AMORIM LA, et al. Vigilância em Saúde do Trabalhador na Atenção Básica: aprendizagens com as equipes de Saúde da Família de João Pessoa, Paraíba, Brasil. Revista Ciência \& Saúde Coletiva, 2017; 22(10): 3403-3413.

5. AREHART CH, et al. Tracking U.S. Pertussis Incidence: Correlation of Public Health Surveillance and Google Search Data Varies by State. Scientific Republic, 2019; 9: 19801.

6. BOULIERI A, et al. A Bayesian mixture modeling approach for public health surveillance. Biostatistics, 2018; 21(3): 369-383.

7. BRASIL. Congresso Nacional. Lei no 8080.1990. Disponível em: http://www.planalto.gov.br/ccivil_03/leis//8080.htm. Acessado em: 27 de abril de 2021.

8. BRASIL. Conselho Nacional de Saúde. Resolução no 588.2018. Disponível em: http://conselho.saude.gov.br/resolucoes/2018/Res0588.pdf. Acessado em: 27 de abril de 2021. 
9. BRASIL. Constituição da República Federativa do Brasil. 1988. Disponível em: http://www.planalto.gov.br/ccivil_03/Constituicao/ConstituicaoCompilado.htm. Acessado em: 27 de abril de 2021.

10. BRASIL. Ministério da Saúde. Portaria no 1823.2012 .2 Disponível em: http://bvsms.saude.gov.br/bvs/saudelegis/gm/2012/prt1823_23_08_2012.html. Acessado em: 27 de abril de 2021.

11. BRASIL. Presidência da República. Decreto no 7508.2011 .2 Disponível em: http://www.planalto.gov.br/ccivil_03/_Ato2011-2014/2011/Decreto/D7508.htm. Acessado em: 27 de abril de 2021.

12. CENTERS FOR DISEASE CONTROL AND PREVENTION. Public Health surveillance at CDC: Service through Surveillance. 2018.

13. COSTA GRT, et al. Atuação da Vigilância Ambiental em saúde no controle da Leishmaniose visceral em condomínio horizontal na Região Administrativa Jardim Botânico, Distrito Federal. Revista Comunicação em Ciências da Saúde, 2016; 27(2): 167-172.

14. COSTA ILOF, et al. A vigilância em saúde e o planejamento nas equipes de atenção primária em saúde: revisão narrativa, 2020; (53), e3622.

15. DANTAS AA, SOUZA MKB. Contribuições da residência em planejamento e gestão em saúde no âmbito distrital da vigilância sanitária. Revista Bahiana de Enfermagem, 2020; 34: e34905.

16. DONATELI C, et al. Avaliação da Vigilância em Saúde na Zona da Mata Mineira, Brasil: das normas à prática. Revista Ciência e Saúde Coletiva, 2017; 44(10): 3439-3455.

17. MATURINO MM, et al. A atuação do SUS na vigilância de ambientes de trabalho: a experiência do Centro Estadual de Referência em Saúde do Trabalhador (Cesat) na Bahia. Revista Brasileira de Saúde Ocupacional, 2018; 43 : e10.

18. MOIMAZ SAS, et al. Vigilância em saúde: fluoretação das águas de abastecimento público em 40 municípios do estado de São Paulo, Brasil. Revista Ciência \& Saúde Coletiva, 2020; 25(7): 2653-2662.

19. NETO JHVS, et al. Sistema dedicado para vigilância em saúde: uma revisão bibliográfica. Revista Brasileira de Inovação Tecnológica em Saúde, 2020; 10(1): 43-59.

20. OLDROYD RA, et al. Identifying Methods for Monitoring Foodborne Illness: Review of Existing Public Health Surveillance Technique. JMIR Public Health and Surveillance, 2018; 4(2): e57.

21. PINTOR EAS, GARBIN AC. Notificações de violência relacionadas ao trabalho e vigilância em saúde do trabalhador: rompendo a invisibilidade. Revista Brasileira de Saúde Ocupacional, 2019; 44: e20.

22. RIVERA LA, et al. Evaluation of the ability of standardized supports to improve public health response to syndromic surveillance for respiratory diseases in Canada. BMC Public Health, 2017; 17: 199.

23. SILVEIRA M, FENNER ALD. Avaliação de Impactos à Saúde (AIS): análises e desafios para a Vigilância em Saúde do Brasil. Revista Ciência \& Saúde Coletiva, 2017; 22(10): 3205-3214.

24. SOUZA GS, et al. Presença de agrotóxicos na atmosfera e risco à saúde humana: uma discussão para a Vigilância em Saúde Ambiental. Revista Ciência \& Saúde Coletiva, 2017; 22(10): 3269-3280.

25. TEIXEIRA MG, et al. Vigilância em Saúde no SUS - construção, efeitos e perspectivas. Revista Ciência \& Saúde Coletiva, 2018; 23(6).

26. VANDENBERG O, et al. Control of Infectious Diseases in the Era of European Clinical Microbiology Laboratory Consolidation: New Challenges and Opportunities for the Patient and for Public Health Surveillance. Frontiers in Medicine, 2018; 5(15): 1-7.

27. YANG L, et al. Evaluating Oregon's occupational public health surveillance system based on the CDC updated guidelines. American Journal of Industrial Medicine, 2020; 63(8): 713-725. 